\title{
Dependência de opioide em pacientes com dor crônica*
}

\section{Opioid dependence in chronic pain patients}

\author{
Daiana Ciléa Honorato Nascimentoํㅜ, Rioko Kimiko Sakata² \\ * Recebido do Departamento de Anestesiologia, Dor e Terapia Intensiva da Universidade Federal de São Paulo. \\ São Paulo, SP.
}

\section{RESUMO}

JUSTIFICATIVA E OBJETIVOS: Apesar dos opioides serem úteis no tratamento da dor crônica não oncológica, há reservas, principalmente devido ao abuso e dependência. O objetivo deste estudo foi rever o uso de opioides em pacientes com dor crônica não oncológica.

CONTEÚDO: Foram descritas as definições de tolerância, abuso e dependência, a incidência do uso de opioide e de dependência em pacientes com dor crônica, os fatores de risco, os sinais de dependência, as estratégias para reduzir o risco e o tratamento da dor em dependente.

CONCLUSÃO: Tanto o diagnóstico, quanto o tratamento dos pacientes dependentes de opioides podem ser difíceis. No entanto, a dor não pode ser subtratada e o médico deve estar atento às alterações que indiquem abuso. Portanto, o conhecimento médico sobre dependência de opioides deve ser acurado para possibilitar a melhor conduta.

Descritores: Dependência, Dor crônica, Opioides, Tratamento da dor.

\section{SUMMARY}

BACKGROUND AND OBJECTIVES: Although opioids are useful to treat chronic non-oncologic pain, there are caveats, especially due to abuse and dependence.

1. Aluna do Curso de Residência Médica em Dor da Universidade Federal de São Paulo (UNIFESP). São Paulo, SP, Brasil. 2. Professora Associada, Coordenadora do Setor de Dor da Universidade Federal de São Paulo. (UNIFESP). São Paulo, SP, Brasil.

Endereço para correspondência:

Dra. Rioko Kimiko Sakata

R. Três de Maio 61/51 - Vila Clementino

04044-020 São Paulo, SP.

Fone: (11) 5576-4069

E-mail: riokoks.dcir@epm.br
This study aimed at reviewing the use of opioids in chronic non-oncologic pain.

CONTENTS: Definitions of tolerance, abuse and dependence, the incidence of opioid use and dependence in chronic pain patients, risk factors, dependence signs, strategies to decrease risk and pain treatment in dependent patients were described.

CONCLUSION: Both diagnosis and treatment of opioid-dependent patients may be difficult. However, pain cannot be subtracted and the physician must be aware of changes indicating abuse. So, the medical understanding about opioids dependence has to be accurate to allow for better management.

Keywords: Chronic pain, Dependence, Opioids, Pain management.

\section{INTRODUÇÃO}

Os opioides são os fármacos de escolha para o alívio da dor aguda e dor oncológica intensa. Também têm sido utilizados no tratamento de diversas síndromes dolorosas crônicas não oncológicas.

Embora seu uso para dor crônica não oncológica seja controverso, há evidências crescentes benéficas em algumas populações de pacientes. O grande temor do uso prolongado de opioides para esses pacientes é que, apesar de promover excelente efeito analgésico, há aumento substancial do risco da principal complicação que é o vício ${ }^{1}$.

Para reduzir o risco e obter melhor efeito dos opioides é necessário que os profissionais administrem o medicamento de forma consciente, com indicações precisas, fazendo avaliações periódicas. $\mathrm{O}$ uso prolongado de opioides provoca inúmeras alterações celulares responsáveis pelo desenvolvimento de três fenômenos clínicos: tolerância, síndrome de abstinência e dependência. 
O objetivo deste estudo foi rever o uso de opioides em pacientes com dor crônica não oncológica.

\section{TOLERÂNCIA, ABUSO E DEPENDÊNCIA}

Conceituar os termos tolerância, pseudodependência, síndrome de dependência e vício torna-se imprescindível para aprimorar o diagnóstico médico e evitar erros no tratamento de pacientes que fazem uso de opioides.

Tolerância é definida como aumento da dose necessária para obter os mesmos efeitos anteriores, ou redução dos efeitos desejados em paciente recebendo a mesma dose. Abuso é o uso de substância psicoativa de maneira imprópria para as convenções psicossociais. O paciente recebe substância controlada de algum local que não o do médico que prescreve no centro onde faz o tratamento da dor, exceto substância controlada para lesão aguda não relacionada ao problema que está sendo tratado ${ }^{2}$.

Síndrome de dependência é um conjunto de fenômenos fisiológicos, comportamentais e cognitivos, de intensidade variável, em que o uso de psicoativos assume uma prioridade ${ }^{3,4}$.

Dependência química é a dependência física e/ou psicológica para uma ou mais substância psicoativa. É uma adaptação à presença contínua do fármaco no organismo, manifestando a síndrome de abstinência com a sua retirada abrupta, diminuição da dose ou utilização de antagonista ${ }^{1}$.

Dependência psicológica é a necessidade de substância psicoativa pelo efeito positivo ou para evitar sintomas de abstinência. Vício é uma síndrome psicológica e comportamental caracterizada pela evidência de dependência com uso aberrante de substância psicoativa, perda de controle e uso compulsivo, apesar dos efeitos adversos. Vício de opioides como uma doença neurobiológica, crônica, primária com fatores genéticos, psicossociais, e fatores ambientais influenciando no desenvolvimento e manifestação.

Pseudodependência é o que ocorre com tratamento da dor com doses baixas de opioides, levando o paciente a relatar dor mesmo estando sob medicação.

A síndrome de abstinência é caracterizada pelos sintomas físicos e/ou psicológicos advindos da parada ou redução abrupta da substância. Pode surgir em $24 \mathrm{~h}$ após a administração do opioide, levando a necessidade de aumento da dose utilizada para se obter o mesmo efeito analgésico inicial ${ }^{5}$. A síndrome de abstinência ocorre devido a uma hiperatividade dos neurônios noradrenérgicos situados dentro do locus ceruleus $^{6}$, localizado na região dorso-lateral do teg- mento pontino, é responsável pela maior parte da produção de noradrenalina do sistema nervoso central (SNC), provocando os sintomas de estimulação simpática na síndrome de abstinência. Os sintomas surgem 15 a 20hs após a última dose, com pico em 2 a 3 dias e remissão em 10 a 14 dias $^{7}$. Os sintomas que surgem com a retirada do opioide incluem aumento da pressão arterial, da frequência cardíaca, midríase, desejo do fármaco, ansiedade, agitação, irritabilidade, sudorese, rinorreia, bocejo, aumento da intensidade da dor, piloereção, anorexia, náusea, vômito, diarreia, cólica abdominal, dor óssea e muscular e tremor ${ }^{1}$.

Para evitar o desenvolvimento da síndrome de abstinência, os opioides devem ser descontinuados, com redução progressiva da dose. A gravidade dos seus sintomas depende da substância envolvida, dose e tempo de uso. A clonidina, é efetiva na supressão dos sintomas adrenérgicos da sindrome de abstinência por diminuir a transmissão das vias simpáticas no SNC.

\section{DOR CRÔNICA E USO DE OPIOIDE}

É evidente o aumento do uso de opioides para tratamento da dor crônica não oncológica nas últimas décadas. Também surgiram controvérsias se esse medicamento causa mais lesão que benefício ${ }^{8-10}$. Não existe dado muito forte para apoiar o uso de opioides em longo prazo para alívio da dor ${ }^{12}$.

Cerca de $30 \%$ dos indivíduos tem dor crônica e destes, $10 \%$ são tratados com opioides ${ }^{12}$. A dor não oncológica representa de $60 \%$ a $70 \%$ dos casos de dor crônica e muitas vezes outros medicamentos não promovem efeito analgésico. Para estes pacientes, porém, o tratamento com opioides deve ser bastante criterioso ${ }^{13}$. Entretanto, o seu uso antes do paciente ser atendido em centros de dor é maior que $90 \%$.

Grandes doses de opioides são usadas por cerca de 2,5\% de todos os pacientes com dor crônica, especialmente nos com maior número de síndromes dolorosas, desordens clínicas, psiquiátricas e uso de substâncias ${ }^{14}$.

$\mathrm{O}$ valor do uso prolongado de opioide para dor crônica não oncológica é determinado pelo balanço entre benefícios (melhor controle da dor) e riscos (dependência, mau uso, comportamento aberrante) $)^{15}$.

Há vários conceitos errados em relação a prescrição de opioides pelos profissionais ${ }^{17}$. Para que sejam atingidos bons resultados é importante o conhecimento adequado sobre a indicação e os cuidados para sua manutenção. 


\section{DEPENDÊNCIA DE OPIOIDE}

É importante lembrar que a terapia crônica com opioide pode causar dependência ${ }^{12}$. O diagnóstico de abuso e dependência é feito em minoria dos pacientes que estão utilizando opioides, mas isso pode estar sendo subestimado ${ }^{17}$. Somente dados limitados estão disponíveis ${ }^{18}$.

Abuso pode ocorrer em até um terço dos pacientes com uso de opioides ${ }^{2}$. Pode ocorrer por automedicação para dor ou para sintomas não dolorosos, e para obter euforia. Ocorre dependência em até cerca de $25 \%$ dos indivíduos que utilizam opioides ${ }^{17,19,20}$. É importante salientar que em um estudo a dependência a opioide ocorreu por prescrição legal em $30 \%$ a $40 \%{ }^{21,22}$. Após tornarem-se dependentes os indivíduos obtêm também opioide de fonte ilícita ${ }^{21}$.

Deve ser feita prescrição responsável para o tratamento da dor para reduzir a possibilidade de abuso e dependên$\mathrm{cia}^{23}$. A dependência é mais frequente com opioide de ação e pico rápidos, e que causam mais sedação e euforia. Os de liberação rápida apresentam maior potencial euforizante que os de liberação lenta, e, portanto, maior risco de dependência ${ }^{24}$.

Os indivíduos com doença psiquiátrica e desordem de uso de substâncias apresentam aumento do risco. O efeito da desordem de uso de substância é mais intenso, apesar da doença mental ser mais frequente. O risco é menor com menor dose durante curto tempo.

Uma grande parte dos opioides é obtida de amigos e familiares. Outros meios são: prescrição por vários profissionais, tráfico, fraude na prescrição, furto e aquisição pela internet ${ }^{25}$

\section{FATORES DE RISCO PARA DEPENDÊNCIA}

Os fatores de risco para dependência entre pacientes em tratamento com opioides são: jovens; dor crônica após acidente automobilístico; múltiplas regiões dolorosas; antecedente de uso de drogas ilícitas; depressão, doença psiquiátrica; uso de medicamento psicotrópico; dependência de tabaco; dose maior; maior tempo de uso; uso de álcool e uso por familiar ${ }^{2,17,19,20}$.

Há relação inversa entre idade e diagnóstico de abuso. $\mathrm{O}$ efeito da desordem do uso de substâncias é especialmente forte ${ }^{17}$.

Acredita-se que a dependência ocorra devido a uma combinação de fatores que incluem a predisposição genética, perfil psicológico, contexto sócio-cultural e exposição ao fármaco.

No contexto do tratamento da dor crônica com opioide, o fator mais importante para o risco de vício é o histórico pessoal ou familiar de abuso de substância. Tanto os pacientes com história de abuso de drogas e álcool, quanto os com doença psiquiátrica prévia, estão associados com maior desvio de medicamentos. Outras variáveis, como sexo e estado civil, podem contribuir para o risco, mas a correlação é menos evidente ${ }^{1}$.

Os dependentes de opioide muitas vezes fazem uso de substância ilícita como maconha, cocaína, benzodiazepínico, nicotina e álcool ${ }^{2,12,26}$. O uso de droga ilícita também é feito por $16 \%$ de pacientes com dor crônica ${ }^{2}$.

\section{ASPECTOS NEUROBIOLÓGICOS DA DEPENDÊNCIA}

A dependência é uma doença neurobiológica crônica produzida por repetidas exposições e caracterizada pela perda do controle sobre o uso do fármaco. $\mathrm{O}$ uso de substâncias psicotrópicas pode levar a anormalidades do funcionamento cerebral, com desenvolvimento de características básicas da dependência química, tais como a compulsão e a síndrome de abstinência. $\mathrm{O}$ aumento de dopamina no sistema mesolímbico desencadeado pelo uso de opioides, com consequente aumento da transmissão dopaminérgica é considerado primordial para o desenvolvimento de dependência ${ }^{3}$. Os opioides induzem liberação de dopamina indiretamente através da diminuição da inibição de GABA através dos receptores opioides na aréa tegumentar ventral, bem como interagindo com receptores opioides no núcleo accumbens ${ }^{27}$.

\section{DIAGNÓSTICO DA SÍNDROME DE DEPENDÊNCIA}

O diagnóstico preciso de dependência de opioide não é fácil ${ }^{1}$. As manifestações são a preocupação para obter e usar a substância e um persistente comportamento de busca, com a presença de um forte desejo ou compulsão para conseguir o fármaco. Observa-se também o uso compulsivo e continuado apesar das consequências nocivas $^{3,4}$.

A suspeita de dependência deve ser feita em indivíduos com dor crônica e uso prolongado de opioide, que faz uso abusivo de medicamentos, em recuperação de dependência, usou opioide recentemente, com marcas de agulha, trombose na veia e abscesso. Em pacientes internados por trauma, a dependência é mais frequente que nas outras condições clínicas. Entre os dependentes é comum a ocorrência de visitas a serviço de emergência para obter receitas ${ }^{10}$.

São manifestações de dependência a opioides: uso exces- 
sivo de opioide; exagero na dor; etiologia da dor não esclarecida; desejo intenso e preocupação sobre a disponibilidade contínua de opioide; uso compulsivo, caracterizado por: aumento da dose, uso apesar dos efeitos colaterais, uso para tratar sintomas que não são alvo da terapia, ou uso mesmo durante os períodos assintomáticos; comportamentos como: manipulação do médico para a obtenção de opioide adicional, solicitação de receitas precocemente, aquisição de medicamentos de vários médicos ou fontes não médicas, vários telefonemas e visitas a médicos para obter opioide, visita a sala de emergências, venda de opioide, uso de outras substâncias (álcool ou sedativos ou hipnóticos); não aceitação de mudanças no tratamento; não aceitação da prescrição do médico e alteração de comportamento ${ }^{27,28}$.

\section{ENFOQUES AO TRATAMENTO DE DEPENDÊN- CIA DE OPIOIDES}

A presença de dor crônica associada com resposta inadequada ao tratamento de dependência ${ }^{29}$. Para tratar pacientes com dor crônica e dependência de opioide geralmente é feita a troca por metadona ou buprenorfina ${ }^{30,31}$. Além disso, o paciente deve receber tratamento multidisciplinar para a dependência de opioides e dor crônica, que incluem psicoterapias, grupos de autoajuda mútua, e terapia cognitivo comportamental.

A metadona é eficaz, porém pode causar eventos adversos graves que limitam o seu uso ${ }^{31}$. É usada para evitar a síndrome de abstinência e bloquear os efeitos de euforia do consumo de opioides. Entretanto, pode causar arritmia ventricular e aumento de morte associada a esse opioide, com controvérsias quanto a seu uso ${ }^{32}$. Geralmente, ocorre óbito quando há vários fatores de risco, incluindo grandes doses de metadona, outros medicamentos que prolongam o intervalo da onda QT e alterações eletrolíticas. É importante ressaltar que pode ocorrer dependência à metadona.

A buprenorfina é um agonista parcial mu, bem tolerado, utilizado no tratamento de manutenção, com baixo risco de sobredose e de abuso. A dissociação a partir dos receptores é lenta com menos sinais e sintomas de abstinência em relação a outros opioides. A metadona e a buprenorfina podem ter risco de interações potencialmente tóxicas ${ }^{26}$.

\section{ESTRATÉGIAS PARA MELHORAR A DOR MI- NIMIZANDO A POSSIBILIDADE DE ABUSO DE OPIOIDES ${ }^{33}$}

Precaução; avaliar cuidadosamente os pacientes de risco para abuso; selecionar a terapia opioide mais apropriada; monitorar regularmente o paciente para avaliar a eficácia e tolerabilidade do tratamento e detectar possíveis comportamentos aberrantes; ter soluções se observar abuso ou vício ou em caso de falha de tratamento; avaliação periódica; usar opioide de longa duração; dosagem urinária e novas formulações de opioide para impedir ou deter o abuso.

\section{Conduta para paciente dependente de opioide para dor pós-operatória}

Os pacientes com dor crônica e que recebem opioide apresentam dor aguda pós-operatória mais intensa e mais complicações relacionadas com opioide ${ }^{12}$.

$\mathrm{Na}$ manhã da cirurgia, ou na indução da anestesia ou durante o procedimento cirúrgico o dependente em opioide deve receber dose regular de opioide. Deve ser lembrado que o opioide provoca aumento do tempo de esvaziamento gástrico e diminuição da motilidade intestinal. A metadona em altas doses $(>200 \mathrm{mg} / \mathrm{d})$ pode causar arritmia ventricular e aumento do intervalo da onda $\mathrm{QT}^{12}$. Deve ser feita analgesia multimodal com bloqueios e outros analgésicos como anti-inflamatórios não esteroides, cetamina, clonidina ou dexmedetomidina. A analgesia regional deve ser feita sempre que for possível ${ }^{12}$.

Ao passar da via oral para venosa, reduzir a dose principalmente dos opioides com baixa biodisponibilidade como morfina (contrário de metadona e oxicodona) ${ }^{12}$. A reabilitação e desintoxicação não são feitas no período pós-operatório imediato ${ }^{12}$.

\section{Princípios importantes}

Identificação do dependente; prevenção de manifestações de abstinência; verificar a dose necessária de opioide; tratamento dos sintomas psicológicos e tratamento efetivo da dor.

\section{TRATAMENTO DA DOR CRÔNICA EM DEPEN- DENTES DE OPIOIDES}

Em pacientes com dor crônica não oncológica, o tratamento com opioides deve ser por um curto prazo. A decisão de prosseguir com tratamento prolongado com opioide deve ser feita após uma análise cuidadosa do efeito terapêutico obtido, da presença e intensidade dos efeitos adversos, das alterações psiquiátricas que o paciente apresenta e da identificação de comportamentos aberrantes ${ }^{34}$.

Em pacientes de alto risco para uso de opioides, com suspeita de comportamento aberrante, com problemas 
psicossociais ou história de abuso de substâncias, é recomendado o uso apenas se os potenciais riscos podem ser minimizados. Estratégias sugeridas incluem a implementação de uma monitoração frequente e intensa, a prescrição de quantidades limitadas e consulta com profissionais que tenham experiência no tratamento da dependência ou problemas psiquiátricos ${ }^{34,35}$.

Para pacientes com dor crônica e suspeita de desvio de opioide, porém que não sejam dependentes de outras substâncias e que não adquirem os opioides de outras fontes, uma terapia de opioide pode ser indicada. O tratamento da dor crônica deve ser revisto com o paciente e assinado, sendo bem controlado o tipo e dose de opioide, a frequência de distribuição da medicação e dos testes de urina. Para os pacientes que frequentemente usam mais medicamento que o calculado, os opioides devem ser fornecidos diariamente, semanalmente ou quinzenalmente. A frequência do exame de urina deve ser obtida de acordo com os padrões de mau uso e da confiabilidade de seus autorrelatos ${ }^{36}$.

Pacientes com suspeita de abusar de um opioide específico deve, em geral, ser transferido para um opioide diferente. Oxicodona e hidromorfona devem ser usadas com cautela. Outra opção é tentar a redução gradual da dose, caso o paciente utiliza doses maiores que o equivalente a $300 \mathrm{mg} /$ dia de morfina, reduzindo, assim, o ciclo de intoxicação e abstinência desses pacientes. A redução deve ser feita com opioides de liberação controlada, reduzindo-se inicialmente, $10 \%$ a cada 2 a 4 semanas, e após atingir um terço da dose inicial, a redução deve ser de 5\%. A dose ideal será alcançada quando ocorrer manifestação de abstinência ou for atingida uma dose que proporciona analgesia efetiva com sintomas mínimos de abstinência. Pacientes que apresentarem falha com esse tratamento teste após 1 a 3 meses devem ser encaminhados para tratamento com metadona ou buprenorfina $^{36}$.

Devem ser empregados protocolos para reduzir a intensidade da dor, evitando dose excessiva de opioide, com avaliação rigorosa, inclusive da quantidade de medicamento. A escolha dos medicamentos e técnicas analgésicas, dose, via e duração do tratamento deve ser individualizada.

Com seleção cuidadosa de pacientes, prescrição meticulosa e protocolo monitorado, os pacientes com dor não oncológica com possibilidade de obter benefício com opioides não devem ser impedidos de obter esse tratamento $^{11}$. Individualizar escolha da medicação, dose, via, e duração do tratamento ${ }^{12}$.

\section{CONCLUSÃO}

O controle da dor crônica muitas vezes é obtido somente com a utilização de opioide em associação com outros medicamentos. Tanto o diagnóstico, quanto o tratamento dos pacientes dependentes de opioides costumam ser difíceis e as recaídas durante o tratamento da dependência são frequentes, porém a dor não pode ser subtratada e o médico deve estar atento às alterações comportamentais que indiquem abuso. Portanto, o conhecimento médico sobre dependência de opioides deve ser acurado para possibilitar uma melhor conduta.

\section{REFERÊNCIAS}

1. Højsted J, Sjøgren P. Addiction to opioids in chronic pain patients: a literature review. Eur J Pain 2007;11(5):490-518.

2. Manchikanti L, Cash KA, Damron KS, et al. Controlled substance abuse and illicit drug use in chronic pain patients: An evaluation of multiple variables. Pain Physician 2006;9(3):215-25.

3 . Heit HA. The truth about pain management: the difference between a pain patient and an addicted patient. Eur J Pain 2001;5(Suppl A):27-9.

4. Strain EC. Assessment and treatment of comorbid psychiatric disorders in psychiatric disorders in opioid-dependent patients. Clin J Pain 2002;18(4 Suppl):S14-27.

5. Duarte DF. Uma breve história do ópio e dos opioides. Rev Bras Anestesiol 2005;55(1):135-46.

6. Banning A, Sjøgren P, Henriksen H. Pain causes in 200 patients referred to a multidisciplinary cancer pain clinic. Pain 1991;45(1):45-8.

7. Melzack R, Katz J. Pain measurement in persons in pain. In: Wall PD, Melzack R, (editors). Textbook of pain. 4th ed. Edinburgh: Churchil Livingstone; 2000. p. 410-8.

8. Baltiere DA, Andrade AG. Dor e síndrome de dependência de opioide. Rev Bras Med 2008;65(7):223-8.

9. Schneider JP, Kirsh KL. Defining clinical issues around tolerance, hyperalgesia, and addiction: a quantitative and qualitative outcome study of long-term opioid dosing in a chronic pain practice. J Opioid Manag 2010;6(6):385-95.

10. Manchikanti L, Fellows B, Ailinani H, et al. Therapeutic use, abuse, and nonmedical use of opioids: a ten-year perspective. Pain Physician 2010;13(5):401-35. 11. Fishman SM. Strategies for selecting treatment and mitigating risk in patients with chronic pain. J Clin 
Psychiatry 2011;72(1):e02.

12. Brill S, Ginosar Y, Davidson EM. Perioperative management of chronic pain patients with opioid dependency. Curr Opin Anaesthesiol 2006;19(3):325-31. 13. Http: // portal. saude. gov. br/ portal/ arquivos/pdf/ pcdt_uso_opiaceos_dor_cronica.pdf. Protocolo clínico e diretrizes terapêuticas - uso de opiáceos no alívio da dor crônica - codeína, morfina, metadona.

14. Morasco BJ, Duckart JP, Carr TP, et al. Clinical characteristics of veterans prescribed high doses of opioid medications for chronic non-cancer pain. Pain 2010;151(3):625-32.

15. Sullivan MD, Von Korff M, Banta-Green C, et al. Problems and concerns of patients receiving chronic opioid therapy for chronic non-cancer pain. Pain 2010;149(2):345-53.

16. Wolfert MZ, Gilson AM, Dahl JL, et al. Opioid analgesics for pain control: wisconsin physicians' knowledge, beliefs, attitudes, and prescribing practices. Pain Med 2010;11(3):425-34.

17. Edlund MJ, Martin BC, Fan MY, et al. Risks for opioid abuse and dependence among recipients of chronic opioid therapy: results from the TROUP study. Drug Alcohol Depend 2010;112(1-2):90-8.

18. Morasco BJ, Gritzner S, Lewis L, et al. Systematic review of prevalence, correlates, and treatment outcomes for chronic non-cancer pain in patients with comorbid substance use disorder. Pain 2011;152(3):488-97.

19. Boscarino JA, Rukstalis M, Hoffman SN, et al. Risk factors for drug dependence among out-patients on opioid therapy in a large US health-care system. Addiction 2010;105(10):1776-82.

20. Højsted J, Nielsen PR, Guldstrand SK, et al. Classification and identification of opioid addiction in chronic pain patients. Eur J Pain 2010;14(10):1014-20.

21. Canfield MC, Keller CE, Frydrych LM, et al. Prescription opioid use among patients seeking treatment for opioid dependence. J Addict Med 2010;4(2):108-13.

22. Tsui JI, Herman DS, Kettavong M, et al. Physician introduction to opioids for pain among patients with opioid dependence and depressive symptoms. J Subst Abuse Treat 2010;39(4):378-83.

23. Gilson AM. The concept of addiction in law and regulatory policy related to pain management: a critical review. Clin J Pain 2010;26(1):70-7.

24. Ribeiro S, Schimidt AP, Schimidt SRG - O uso de opioides no tratamento da dor crônica não oncológica: o papel da metadona. Rev Bras Anestesiol
2002;52(5):644-51.

25. Fischer B, Bibby M, Bouchard M. The global diversion of pharmaceutical drugs non-medical use and diversion of psychotropic prescription drugs in North America: a review of sourcing routes and control measures. Addiction 2010;105(12):2062-70.

26. McCance-Katz EF, Sullivan LE, Nallani S. Drug interactions of clinical importance among the opioids, methadone and buprenorphine, and other frequently prescribed medications: a review. Am J Addict 2010;19(1):4-16.

27. Ballantyne JC, LaForge KS. Opioid dependence and addiction during opioid treatment of chronic pain. Pain 2007;129(3):235-55.

28. Trescot AM, Boswell MV, Atluri SL, et al. Opioid guidelines in the management of chronic non-cancer-pain. Pain Physician 2006;9(1):1-39.

29. Clark MR, Stoller KB, Brooner RK. Assessment and management of chronic pain in individuals seeking treatment for opioid dependence disorder. Can J Psychiatry 2008;53(8):496-508.

30. Johnson RE, Fudala PJ, Payne R. Buprenorphine: considerations for pain management. J Pain Symptom Manage 2005;29(3):297-326.

31. Rhodin A, Grnbladh L, Nilsson LH, et al. Methadone treatment of chronic non-malignant pain and opioid dependence--a long-term follow-up. Eur J Pain 2006;10(3):271-8.

32. Modesto-Lowe V, Brooks D, Petry N. Methadone deaths: risk factors in pain and addicted populations. J Gen Intern Med 2010;25(4):305-9.

33. Raffa RB, Pergolizzi JV Jr - Opioid formulations designed to resist/deter abuse. Drugs 2010;70(13):165775.

34. Chou R, Clark E, Helfand M. Comparative efficacy and safety of long acting oral opioids for chronic non cancer pain: a systematic review, J Pain Symptom Manage 2003;26(5):1026-48.

35. Chou R, Fanciullo G, Fine P, et al. Clinical guidelines for the use of chronic opioid therapy in chronic noncancer pain. J Pain 2009;10(2):113 30.

36. Kahan M, Srivastava A, Wilson L, et al. Misuse of and dependence on opioids: study of chronic pain patients. Can Fam Physician 2006;52(9):1081-7.

Apresentado em 15 de janeiro de 2011. Aceito para publicação em 02 de junho de 2011. 